\title{
SUFFICIENT GLOBAL OPTIMALITY CONDITIONS FOR MULTI-EXTREMAL SMOOTH MINIMISATION PROBLEMS WITH BOUNDS AND LINEAR MATRIX INEQUALITY CONSTRAINTS
}

\author{
N. Q. HUY 1 , V. JEYAKUMAR ${ }^{ø 2}$ and G. M. LEE ${ }^{3}$
}

(Received 10 February, 2006; revised 1 March, 2006)

\begin{abstract}
In this paper, we present sufficient conditions for global optimality of a general nonconvex smooth minimisation model problem involving linear matrix inequality constraints with bounds on the variables. The linear matrix inequality constraints are also known as "semidefinite" constraints which arise in many applications, especially in control system analysis and design. Due to the presence of nonconvex objective functions, such minimisation problems generally have many local minimisers which are not global minimisers. We develop conditions for identifying global minimisers of the model problem by first constructing a (weighted sum of squares) quadratic underestimator for the twice continuously differentiable objective function of the minimisation problem and then by characterising global minimisers of the easily tractable underestimator over the same feasible region of the original problem. We apply the results to obtain global optimality conditions for optimisation problems with discrete constraints.
\end{abstract}

2000 Mathematics subject classification: primary 41 A65, 41 A29; secondary 90C30.

Keywords and phrases: smooth nonconvex minimisation, global optimality conditions, box constraints, discrete constraints, linear matrix inequalities, multi-extremal problems.

\section{Introduction}

Consider the following nonconvex smooth optimisation model problem:

$$
\min _{x \in \mathbb{R}^{n}} f(x) \text { s.t. }\left\{\begin{array}{l}
F_{0}+\sum_{i=1}^{n} x_{i} F_{i} \succeq 0, \\
x \in \prod_{i=1}^{n}\left[u_{i}, v_{i}\right],
\end{array}\right.
$$

\footnotetext{
'Department of Mathematics, Hanoi Pedagogical University No. 2, Vinh Phuc, Vietnam. ${ }^{2}$ Department of Applied Mathematics, University of New South Wales, Sydney NSW 2052, Australia; e-mail: jeya@maths.unsw.edu.au.

${ }^{3}$ Department of Applied Mathematics, Pukyong National University, Pusan 608-737, Korea; e-mail: gmlee@pknu.ac.kr.

(C) Australian Mathematical Society 2006, Serial-fee code 1446-1811/06
} 
where $f: \mathbb{R}^{n} \rightarrow \mathbb{R}$ is a twice continuously differentiable function, $u_{i}, v_{i} \in \mathbb{R}$ and $u_{i} \leq v_{i}, i=1, \ldots, n, F_{i} \in S^{m}, i=0,1, \ldots, m$ and $S^{m}$ is the set of all symmetric $m \times m$ matrices. The linear matrix inequality (LMI) constraint, $F_{0}+\sum_{i=1}^{n} x_{i} F_{i} \succeq 0$, means that the matrix $F_{0}+\sum_{i=1}^{n} x_{i} F_{i}$ is positive semidefinite. Optimisation model problems with LMI constraints are also known as semidefinite optimisation problems $[6,11]$. Semidefinite optimisation has now come to be recognised as a valuable numerical as well as a modelling tool for control system analysis and design [3], and for many practical discrete optimisation problems [3,12]. Model problems of type (LMIP ${ }_{0}$ ) cover a large class of nonconvex continuous optimisation problems, including quadratic programming problems which arise in various applications [5]. Moreover, continuous relaxations of many discrete optimisation model problems such as optimisation problems with bivalent constraints [10], where $x_{i}=-1$ or $x_{i}=+1$ or binary constraints [2], where $x_{i}=0$ or $x_{i}=1$, are of the form (LMIP $)$. Such discrete problems include the quadratic assignment problem [10] and the max-cut problem [2], arising in routing and scheduling in the area of transportation management.

Due to the presence of nonconvex objective functions, the problems $\left(\mathrm{LMIP}_{0}\right)$ generally have many local minimisers which are not global minimisers. In recent years, much attention has been focused on developing criteria which identify global minimisers of multi-extremal quadratic optimisation problems [2,7-10]. In this paper we present conditions which guarantee that a given feasible point is a global minimiser of the general nonconvex smooth minimisation problem $\left(\mathrm{LMIP}_{0}\right)$. Our approach to developing a global optimality condition is based on quadratic underestimators [1]. We first show that the objective function admits a (weighted sum of squares) quadratic underestimator. We then characterise global minimisers of the underestimator over the feasible region of the original problem. We finally apply this approach to obtain global optimality criteria for discrete optimisation problems which include problems with bivalent constraints or binary constraints.

The paper is organised as follows. Section 2 presents basic recent results on the characterisations of global solutions of weighted least squares problems. Section 3 develops sufficient global optimality conditions for $\left(\mathrm{LMIP}_{0}\right)$ with the box constraints. Section 4 provides global optimality conditions for $\left(\mathrm{LMIP}_{0}\right)$ with discrete constraints.

\section{Preliminaries: quadratic minimisation problems}

In this section, we present basic results on the characterisations of global solutions of weighted least squares problems subject to box or binary constraints and they play key roles in the development of sufficient global optimality conditions. We begin by presenting basic definitions and notation that will be used throughout the paper. The real line is denoted by $\mathbb{R}$ and the $n$-dimensional Euclidean space is denoted by $\mathbb{R}^{n}$. 
For vectors $x, y \in \mathbb{R}^{n}, x \geq y$ means that $x_{i} \geq y_{i}$, for $i=1, \ldots, n$. The identity matrix is denoted by $I$. The notation $A \succeq 0$ means that the matrix $A$ is positive semi-definite. A diagonal matrix with diagonal elements $\alpha_{1}, \ldots, \alpha_{n}$ is denoted by $\operatorname{diag}\left(\alpha_{1}, \ldots, \alpha_{n}\right)$. We will use the symbol $\nabla f(\bar{x})$ (respectively $\left.\nabla^{2} f(\bar{x})\right)$ to denote the gradient (respectively Hessian) of $f(\cdot)$ at $\bar{x}$. Clearly, for each $x \in \mathbb{R}^{n}, \nabla^{2} f(x) \in S^{n}$, the space of all $(n \times n)$ symmetric matrices. The spaces $S^{n}$ and $S^{m}$ are partially ordered by the Löwner order, that is, for $N_{1}, N_{2} \in S^{l}, N_{1} \succeq N_{2}$ if and only if $N_{1}-N_{2}$ is a positive semi-definite matrix.

Consider the quadratic minimisation problem with box constraints which was recently examined in [9]:

$$
\min _{x \in \mathbb{R}^{n}} \frac{1}{2} \sum_{i=1}^{n} \gamma_{i} x_{i}^{2}+\sum_{i=1}^{n} d_{i} x_{i} \quad \text { s.t. } x \in \prod_{i=1}^{n}\left[u_{i}, v_{i}\right],
$$

where $\gamma_{i}, d_{i} \in \mathbb{R}, u_{i}, v_{i} \in \mathbb{R}$ and $u_{i} \leq v_{i}, i=1, \ldots, n$. Define

$$
\hat{\gamma}_{i}:=\max \left\{0,-\gamma_{i}\right\}= \begin{cases}0 & \text { if } \gamma_{i} \geq 0 \\ -\gamma_{i} & \text { if } \gamma_{i}<0 .\end{cases}
$$

For $\bar{x}=\left(\bar{x}_{1}, \ldots, \bar{x}_{n}\right)^{T} \in S$, define

$$
\hat{\bar{x}}_{i}:= \begin{cases}-1 & \text { if } \bar{x}_{i}=u_{i}, \\ 1 & \text { if } \bar{x}_{i}=v_{i}, \\ d_{i}+\gamma_{i} \bar{x}_{i} & \text { if } \bar{x}_{i} \in\left(u_{i}, v_{i}\right) .\end{cases}
$$

For self containment, we provide a proof outline of the following lemma, which was given in [9]. This lemma plays a key role in developing sufficient optimality conditions for $\left(\mathrm{LMIP}_{0}\right)$.

Proposition 2.1 ([9]). For $\left(\mathrm{QP}_{0}\right)$, let $\bar{x} \in S:=\prod_{i=1}^{n}\left[u_{i}, v_{i}\right]$. Then $\bar{x}$ is a global minimiser of $\left(\mathrm{QP}_{0}\right)$ if and only if, for each $i=1, \ldots, n$,

$$
\frac{1}{2} \hat{\gamma}_{i}\left(v_{i}-u_{i}\right)+\hat{\bar{x}}_{i}\left(d_{i}+\gamma_{i} \bar{x}_{i}\right) \leq 0 .
$$

Proof. Let $f(x):=(1 / 2) \sum_{i=1}^{n} \gamma_{i} x_{i}^{2}+\sum_{i=1}^{n} d_{i} x_{i}$, for $x \in \mathbb{R}^{n}$. By definition, $\bar{x}$ is a global minimiser of $\left(\mathrm{QP}_{0}\right)$ if and only if, for each $x \in S$,

$$
\begin{aligned}
f(x)-f(\bar{x}) & =\frac{1}{2} \sum_{i=1}^{n} \gamma_{i} x_{i}^{2}+\sum_{i=1}^{n} d_{i} x_{i}-\left[\frac{1}{2} \sum_{i=1}^{n} \gamma_{i} \bar{x}_{i}^{2}+\sum_{i=1}^{n} d_{i} \bar{x}_{i}\right] \\
& =\sum_{i=1}^{n} \frac{\gamma_{i}}{2}\left(x_{i}-\bar{x}_{i}\right)^{2}+\sum_{i=1}^{n}\left(d_{i}+\gamma_{i} \bar{x}_{i}\right)\left(x_{i}-\bar{x}_{i}\right) \\
& \geq 0 .
\end{aligned}
$$


Thus $\bar{x}$ is a global minimiser of $\left(\mathrm{QP}_{0}\right)$ if and only if for each $i=1, \ldots, n, x_{i} \in\left[u_{i}, v_{i}\right]$,

$$
\frac{\gamma_{i}}{2}\left(x_{i}-\bar{x}_{i}\right)^{2}+\left(d_{i}+\gamma_{i} \bar{x}_{i}\right)\left(x_{i}-\bar{x}_{i}\right) \geq 0 .
$$

The equivalence of (2.4) and (2.3) is obtained by directly verifying the equivalence in three simple cases, where $\bar{x}_{i}=u_{i}, \bar{x}_{i}=v_{i}$ and $\bar{x}_{i} \in\left(u_{i}, v_{i}\right)$.

Consider the following minimisation problem with discrete constraints:

$$
\min _{x \in \mathbb{R}^{n}} \sum_{i=1}^{n} \frac{1}{2} \gamma_{i} x_{i}^{2}+\sum_{i=1}^{n} d_{i} x_{i} \quad \text { s.t. } x \in \prod_{i=1}^{n}\left\{u_{i}, v_{i}\right\},
$$

where $\gamma_{i}, d_{i} \in \mathbb{R}, u_{i}, v_{i} \in \mathbb{R}$ and $u_{i} \leq v_{i}, i=1, \ldots, n$.

PROPOSITION 2.2 ([9]). For the problem $\left(\mathrm{BQP}_{0}\right)$, let $\bar{x} \in S_{B}:=\prod_{i=1}^{n}\left\{u_{i}, v_{i}\right\}$. Let $\hat{\bar{x}}_{i}$ be defined by (2.2). Then $\bar{x}$ is a global minimiser of $\left(\mathrm{BQP}_{0}\right)$ if and only if for each $i=1, \ldots, n$,

$$
\hat{\bar{x}}_{i}\left(d_{i}+\gamma_{i} \bar{x}_{i}\right)-\frac{\gamma_{i}}{2}\left(v_{i}-u_{i}\right) \leq 0
$$

PROOF. The proof is similar to the proof of Proposition 2.1 and so is omitted.

\section{Smooth minimisation with box constraints}

In this section, we derive sufficient global optimality conditions for smooth minimisation problems with box constraints. We consider the problem, discussed in the Introduction:

$$
\min _{x \in \mathbb{R}^{n}} f(x) \text { s.t. }\left\{\begin{array}{l}
F_{0}+\sum_{i=1}^{n} x_{i} F_{i} \succeq 0, \\
x \in \prod_{i=1}^{n}\left[u_{i}, v_{i}\right],
\end{array}\right.
$$

where $f: \mathbb{R}^{n} \rightarrow \mathbb{R}$ is a twice continuously differentiable function on an open set containing $\Delta:=\prod_{i=1}^{m}\left[u_{i}, v_{i}\right], F_{i} \in S^{m}, i=0, \ldots, n$ and $S^{m}$ is the space of all symmetric $m \times m$ matrices. Let $S_{+}^{m}=\left\{M \in S^{m} \mid M \succeq 0\right\}$ and let $\Gamma=\left\{x \in \mathbb{R}^{n} \mid\right.$ $\left.F_{0}+\sum_{i=1}^{n} x_{i} F_{i} \in S_{+}^{m}\right\}$. Set $D:=\Gamma \cap \Delta$.

For each $\bar{x}=\left(\bar{x}_{1}, \ldots, \bar{x}_{2}\right)^{T} \in D$, the gradient and the Hessian are given by

$$
\nabla f(\bar{x})=\left(\frac{\partial f}{\partial x_{1}}(\bar{x}), \ldots, \frac{\partial f}{\partial x_{n}}(\bar{x})\right)^{T} \quad \text { and } \quad \nabla^{2} f(\bar{x})=\left(a_{i j}(\bar{x})\right),
$$



where $a_{i j}(\bar{x})=\partial^{2} f(\bar{x}) / \partial x_{i} \partial x_{j}, i, j=1, \ldots, n$. For each $i=1, \ldots, n$, define

$$
\begin{aligned}
& \alpha_{i}:=\min \left\{a_{i i}(z)-\sum_{j=1, j \neq i}^{n}\left|a_{i j}(z)\right|: z \in \Delta\right\}, \\
& G:=\operatorname{diag}\left(\alpha_{1}, \ldots, \alpha_{n}\right) .
\end{aligned}
$$

We recall that an $n \times n$ symmetric matrix $A:=\left(\gamma_{i j}\right)_{n \times n}$ is said to be diagonally dominant if $\left|\gamma_{i i}\right| \geq \sum_{j=1, j \neq i}^{n}\left|\gamma_{i j}\right|$, for $i=1, \ldots, n$. Every diagonally dominant symmetric matrix $A$ with non-negative diagonal elements is positive semi-definite. For more details we refer the reader to [4].

Let

$$
F(x)=F_{0}+\sum_{i=1}^{n} x_{i} F_{i}, \quad \hat{F}(x)=\sum_{i=1}^{n} x_{i} F_{i}, \quad x=\left(x_{1}, \ldots, x_{n}\right) \in \mathbb{R}^{n} .
$$

Then $\hat{F}(\cdot)$ is a linear operation from $\mathbb{R}^{n}$ to $S^{m}$ and its dual is defined by

$$
\hat{F}^{*}(M)=\left(\operatorname{Tr}\left[F_{1} M\right], \ldots, \operatorname{Tr}\left[F_{n} M\right]\right)^{T} \text { for any } M \in S^{m},
$$

where $\operatorname{Tr}[\cdot]$ is the trace operation. For details, see $[3,12]$.

For (LMIP $)_{0}$, define a quadratic function $g: \mathbb{R}^{n} \rightarrow \mathbb{R}$ by

$$
g(x):=\frac{1}{2} x^{T} G x+\left(\nabla f(\bar{x})-G \bar{x}-\hat{F}^{*}(M)\right)^{T} x,
$$

where $M \in S^{m}$.

Recall that the function $h: \mathbb{R}^{n} \rightarrow \mathbb{R}$ is a quadratic underestimator for the objective function $f$ at $\bar{x}$ over $D$ if $h$ is a quadratic function, and, for each $x \in D, f(x) \geq$ $h(x)$, and $f(\bar{x})=h(\bar{x})$. For applications of quadratic underestimators in numerical optimisation, see [1].

LEMMA 3.1. Let $\bar{x} \in D:=\Gamma \cap \Delta$. Suppose that there exists $M \succeq 0$ such that $\operatorname{Tr}[M F(\bar{x})]=0$. Then,

(i) for each $x \in D, f(x)-f(\bar{x}) \geq g(x)-g(\bar{x})$;

(ii) the function $h: \mathbb{R}^{n} \rightarrow \mathbb{R}$, defined by $h(x)=g(x)-g(\bar{x})+f(\bar{x})$, is a quadratic underestimator of $f$ at $\bar{x}$ over $D$.

PROOF. (i) Let $l(x):=f(x)-\hat{F}^{*}(M)^{T} x-\operatorname{Tr}\left[M F_{0}\right]$ and let $\varphi(x):=l(x)-g(x)$, $x \in \Delta$. It is easy to show that $\nabla \varphi(\bar{x})=0$ and $\nabla^{2} \varphi(z)=\nabla^{2} f(z)-G$ for all $z \in \Delta$. Moreover, for all $z \in \Delta$,

$$
\nabla^{2} \varphi(z)=\left(\gamma_{i j}(z)\right)_{n \times n}=\left(\begin{array}{cccc}
a_{11}(z)-\alpha_{1} & a_{12}(z) & \cdots & a_{1 n}(z) \\
a_{21}(z) & a_{22}(z)-\alpha_{2} & \cdots & a_{2 n}(z) \\
\vdots & \vdots & \ddots & \vdots \\
a_{n 1}(z) & a_{n 2}(z) & \cdots & a_{n n}(z)-\alpha_{n}
\end{array}\right) .
$$


Since $\varphi(\cdot)$ is twice continuously differentiable, $\nabla^{2} \varphi(z) \in S^{n}$ for all $z \in \Delta$. From (3.1) it follows that $a_{i i}(z)-\alpha_{i} \geq \sum_{j=1, j \neq i}^{n}\left|a_{i j}(z)\right| \geq 0$ for all $z \in \Delta$ and $i=1, \ldots, n$. So, for each $z \in \Delta$, the matrix $\nabla^{2} \varphi(z)$ is diagonally dominant with non-negative diagonal elements. Hence $\nabla^{2} \varphi(z) \in S_{+}^{m}$ for every $z \in \Delta$. Since for each $x \in \Delta$, there is $z \in \Delta$ such that

$$
\varphi(x)-\varphi(\bar{x})=\nabla \varphi(\bar{x})^{T}(x-\bar{x})+\frac{1}{2}(x-\bar{x})^{T} \nabla^{2} \varphi(z)(x-\bar{x}),
$$

it follows that $\varphi(x)-\varphi(\bar{x}) \geq 0$ for all $x \in \Delta$. Therefore $l(x)-l(\bar{x}) \geq g(x)-g(\bar{x})$ for all $x \in \Delta$, and hence $l(x)-l(\bar{x}) \geq g(x)-g(\bar{x})$ for all $x \in D$. Since $M \in S_{+}^{m}$ and $F(x) \in S_{+}^{m}$, for all $x \in \Gamma$, we have $\operatorname{Tr}[M F(x)] \geq 0$, for all $x \in D \subset \Gamma$. Hence

$$
\begin{aligned}
f(x)-f(\bar{x}) & \geq f(x)-\operatorname{Tr}[M F(x)]-f(\bar{x}) \\
& =f(x)-\operatorname{Tr}[M F(x)]-(f(\bar{x})-\operatorname{Tr}[M F(\bar{x})]) \\
& =l(x)-l(\bar{x}) \\
& \geq g(x)-g(\bar{x}) \quad \forall x \in D .
\end{aligned}
$$

(ii) The conclusion follows from (i) since, for each $x \in D, f(x) \geq h(x)$ and $f(\bar{x})=h(\bar{x})$.

LEMma 3.2. Let $\bar{x} \in D:=\Gamma \cap \Delta$. Suppose that there exists $M \succeq 0$ such that $\operatorname{Tr}[M F(\bar{x})]=0$. If $\bar{x}$ is a global minimiser of the quadratic function $g$ over $\Delta$, then $\bar{x}$ is a global minimiser of $\left(\mathrm{LMIP}_{0}\right)$.

PRoOF. Since $\bar{x}$ minimises $g(\cdot)$ over $\Delta, \bar{x}$ minimises $g(\cdot)$ over $D$, that is, $g(x)-$ $g(\bar{x}) \geq 0$ for all $x \in D$. Now, it follows from Lemma 3.1 that $\bar{x}$ is a global minimiser of (LMIP $)$.

For $\left(\mathrm{LMIP}_{0}\right)$, we assume, without loss of generality, that $u_{i}<v_{i}, i=1, \ldots, n$. For each $i=1, \ldots, n$, define

$$
\begin{aligned}
& \hat{\alpha}_{i}:=\max \left(0,-\alpha_{i}\right), \\
& \hat{G}:=\operatorname{diag}\left(\hat{\alpha}_{1}, \ldots, \hat{\alpha}_{n}\right) .
\end{aligned}
$$

Let $u=\left(u_{1}, \ldots, u_{n}\right)^{T}$ and $v=\left(v_{1}, \ldots, v_{n}\right)^{T}$. Let $\bar{x}=\left(\bar{x}_{1}, \ldots, \bar{x}_{n}\right)^{T} \in D$. Define

$$
\tilde{x}_{i}:= \begin{cases}-1 & \text { if } \bar{x}_{i}=u_{i}, \\ 1 & \text { if } \bar{x}_{i}=v_{i}, \\ \left(\nabla f(\bar{x})-\hat{F}^{*}(M)\right)_{i} & \text { if } \bar{x}_{i} \in\left(u_{i}, v_{i}\right),\end{cases}
$$

and

$$
\tilde{X}:=\operatorname{diag}\left(\tilde{x}_{1}, \ldots, \tilde{x}_{n}\right)
$$


THEOREM 3.3. Let $\bar{x} \in D:=\Gamma \cap \Delta$. If there exists $M \succeq 0$ such that $\operatorname{Tr}[M F(\bar{x})]=0$ and

$$
\frac{1}{2} \hat{G}(v-u)+\tilde{X}\left(\nabla f(\bar{x})-\hat{F}^{*}(M)\right) \leq 0,
$$

then $\bar{x}$ is a global minimiser of $\left(\mathrm{LMIP}_{0}\right)$.

PROOF. Let

$$
g(x):=\frac{1}{2} x^{T} G x+\left(\nabla f(\bar{x})-G \bar{x}-\hat{F}^{*}(M)\right)^{T} x \quad \text { and } \quad \Delta:=\prod_{i=1}^{n}\left[u_{i}, v_{i}\right] .
$$

The conclusion will follow from Lemma 3.2 if we show that $\bar{x}$ is a global minimiser of $g$ over $\Delta$. Since $G=\operatorname{diag}\left(\alpha_{1}, \ldots, \alpha_{n}\right)$ and $d_{i}=(\nabla f(\bar{x}))_{i}-\alpha_{i} \bar{x}_{i}-\left(\hat{F}^{*}(M)\right)_{i}$, it follows from Proposition 2.1 that $\bar{x}$ is a global minimiser of $g$ over $\Delta$ if and only if for each $i=1, \ldots, n, \frac{1}{2} \hat{\alpha}_{i}\left(v_{i}-u_{i}\right)+\tilde{x}_{i}\left((\nabla f(\bar{x}))_{i}-\left(\hat{F}^{*}(M)\right)_{i}\right) \leq 0$. That is, $\frac{1}{2} \hat{G}(v-u)+\tilde{X}\left(\nabla f(\bar{x})-\hat{F}^{*}(M)\right) \leq 0$. Now, by the hypothesis, $\bar{x}$ is a global minimiser of $g$ over $\Delta$.

The following simple numerical examples illustrate how global minimisers of smooth minimisation problems can be identified by Theorem 3.3. In the first example a global minimiser occurs at an interior point, whereas in the second example it occurs at a boundary of the feasible set.

EXAMPLE 1. Consider the following smooth minimisation problem:

$$
\min _{x \in \mathbb{R}^{2}} f(x)=x_{1}^{3}+x_{2}^{3}-x_{1}^{2}-x_{2}^{2} \text { s.t. }\left\{\begin{array}{l}
F_{0}+\sum_{i=1}^{2} x_{i} F_{i} \succeq 0, \\
x \in \Delta:=\prod_{i=1}^{2}[1 / 2,11 / 2],
\end{array}\right.
$$

where

$$
F_{0}=\left(\begin{array}{lll}
3 & 1 & 0 \\
1 & 1 & 0 \\
0 & 0 & 1
\end{array}\right), \quad F_{1}=\left(\begin{array}{lll}
0 & 1 & 0 \\
1 & 0 & 0 \\
0 & 0 & 1
\end{array}\right) \quad \text { and } \quad F_{2}=\left(\begin{array}{lll}
1 & 0 & 0 \\
0 & 0 & 0 \\
0 & 0 & 0
\end{array}\right) .
$$

Let $\bar{x}=\left(\bar{x}_{1}, \bar{x}_{2}\right)=(2 / 3,2 / 3) \in D:=\left\{x \in \Delta \mid\left(x_{1}+1\right)^{2} \leq x_{2}+3\right\}$. It is easy to check that $\bar{x}$ is a local minimiser of (E1). Let $z \in \Delta$. Then

$$
\begin{gathered}
F(\bar{x})=\left(\begin{array}{ccc}
11 / 3 & 5 / 3 & 0 \\
5 / 3 & 1 & 0 \\
0 & 0 & 5 / 3
\end{array}\right), \quad \nabla f(\bar{x})=(0,0)^{T} \quad \text { and } \\
\nabla^{2} f(z)=\left(\begin{array}{cc}
6 z_{1}-2 & 0 \\
0 & 6 z_{2}-2
\end{array}\right) .
\end{gathered}
$$


It now follows that $G=\left(\begin{array}{ll}1 & 0 \\ 0 & 1\end{array}\right)$ and $\hat{G}=\left(\begin{array}{ll}0 & 0 \\ 0 & 0\end{array}\right)$. Taking

$$
M=\left(\begin{array}{lll}
0 & 0 & 0 \\
0 & 0 & 0 \\
0 & 0 & 0
\end{array}\right)
$$

we obtain $M \in S_{+}^{3}, \operatorname{Tr}[M F(\bar{x})]=0$ and $\hat{F}^{*}(M)=\left(\operatorname{Tr}\left[M F_{1}\right], \operatorname{Tr}\left[M F_{2}\right]\right)^{T}=(0,0)^{T}$, and so (3.7) holds for (E1) at $\bar{x}=(2 / 3,2 / 3)$. The point $\bar{x}=(2 / 3,2 / 3)$ is a global minimiser of (E1) as is also seen from the graph of $f$ given below

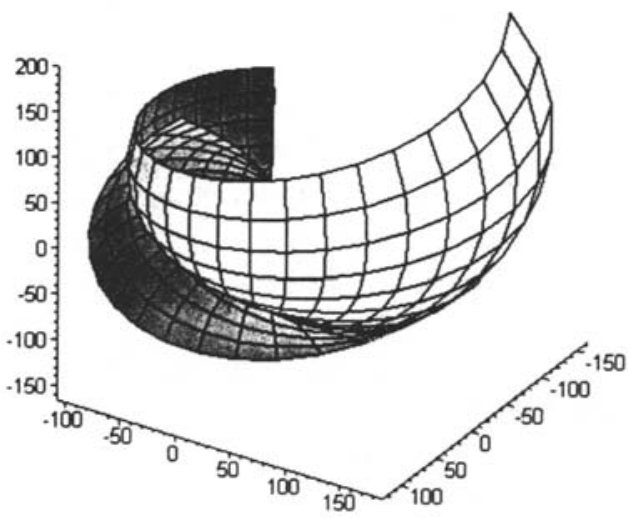

EXAMPLE 2. Consider the following smooth minimisation problem:

$$
\min _{x \in \mathbb{R}^{2}} f(x)=x_{1}^{2} x_{2}+x_{1} x_{2}^{2}-x_{1}-x_{2} \text { s.t. }\left\{\begin{array}{l}
F_{0}+\sum_{i=1}^{2} x_{i} F_{i} \succeq 0, \\
x \in \Delta:=\prod_{i=1}^{2}[-1,0],
\end{array}\right.
$$

where

$$
F_{0}=\left(\begin{array}{lll}
1 & 1 & 0 \\
1 & 1 & 0 \\
0 & 0 & 1
\end{array}\right), \quad F_{1}=\left(\begin{array}{ccc}
0 & -1 & 0 \\
-1 & 0 & 0 \\
0 & 0 & 1
\end{array}\right) \quad \text { and } \quad F_{2}=\left(\begin{array}{lll}
1 & 0 & 0 \\
0 & 0 & 0 \\
0 & 0 & 0
\end{array}\right) \text {. }
$$

Let $\bar{x}=\left(\bar{x}_{1}, \bar{x}_{2}\right)=(0,0) \in D$. Clearly, $\bar{x}$ belongs to the boundary of $D$. Let $z \in \Delta$. Then,

$$
\begin{gathered}
F(\bar{x})=\left(\begin{array}{lll}
1 & 1 & 0 \\
1 & 1 & 0 \\
0 & 0 & 1
\end{array}\right), \quad \nabla f(\bar{x})=(-1,-1)^{T} \text { and } \\
\nabla^{2} f(z)=\left(\begin{array}{cc}
2 z_{2} & 2 z_{1}+2 z_{2} \\
2 z_{1}+2 z_{2} & 2 z_{1}
\end{array}\right) .
\end{gathered}
$$


So, $G=\left(\begin{array}{cc}-6 & 0 \\ 0 & -6\end{array}\right)$ and $\hat{G}=\left(\begin{array}{ll}6 & 0 \\ 0 & 6\end{array}\right)$. Taking

$$
M=\left(\begin{array}{ccc}
2 & -2 & 0 \\
-2 & 2 & 0 \\
0 & 0 & 0
\end{array}\right)
$$

we obtain $M \in S_{+}^{3}, \operatorname{Tr}[M F(\bar{x})]=0$ and $\hat{F}^{*}(M)=\left(\operatorname{Tr}\left[M F_{1}\right], \operatorname{Tr}\left[M F_{2}\right]\right)^{r}=(4,2)^{T}$. Condition (3.7) now becomes

$$
\begin{aligned}
\frac{1}{2} \hat{G}(v-u)+\tilde{X}\left(\nabla f(\bar{x})-\hat{F}^{*}(M)\right) & =\frac{1}{2}\left(\begin{array}{ll}
6 & 0 \\
0 & 6
\end{array}\right)\left(\begin{array}{l}
1 \\
1
\end{array}\right)+\left(\begin{array}{ll}
1 & 0 \\
0 & 1
\end{array}\right)\left(\begin{array}{l}
-5 \\
-3
\end{array}\right) \\
& =\left(\begin{array}{c}
-2 \\
0
\end{array}\right) .
\end{aligned}
$$

Thus (3.7) holds for (E2) at $\bar{x}=(0,0)$ which is a global minimiser of (E2).

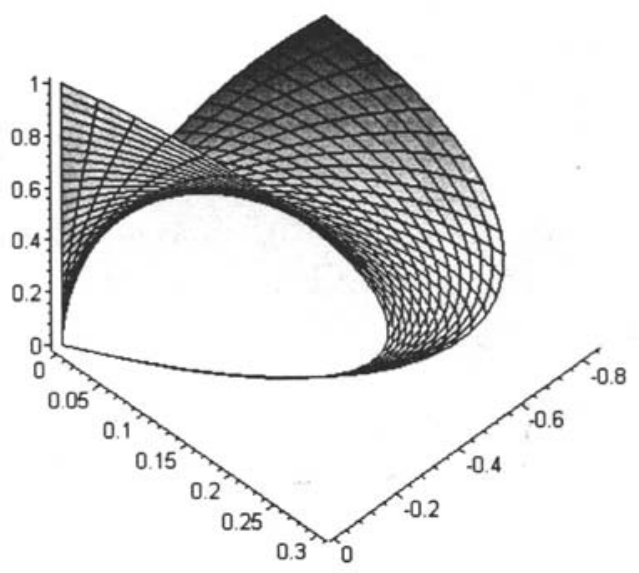

Let us examine a special case of $\left(\mathrm{LMIP}_{1}\right)$ where the matrix inequality constraints are replaced by the standard linear inequalities:

$$
\min _{x \in \mathbb{R}^{n}} f(x) \quad \text { s.t. } \quad\left\{\begin{array}{l}
b_{0}+B x \geq 0, \\
x \in \prod_{i=1}^{n}\left[u_{i}, v_{i}\right],
\end{array}\right.
$$

where $B=\left(b_{i j}\right)_{m \times n}$ is an $m \times n$ matrix and $b_{0}=\left(b_{01}, \ldots, b_{0 m}\right)^{T}$.

COROLLARY 3.4. Let $\bar{x} \in D$. If there exists $\lambda(\geq 0) \in \mathbb{R}^{m}$, such that $\lambda^{T}\left(b_{0}+B x\right)=0$ such that

$$
\frac{1}{2} \hat{G}(v-u)+\tilde{X}(\nabla f(\bar{x})-B \lambda) \leq 0,
$$

then $\bar{x}$ is a global minimiser of $\left(\mathrm{LIP}_{1}\right)$. 
PROOF. For each $i=0, \ldots, n$, let $F_{i}=\operatorname{diag}\left(b_{i 1}, \ldots, b_{i m}\right)$. Let $M=\operatorname{diag}(\lambda)$. Then $\hat{F}^{*}(M)=B \lambda$. Applying Theorem 3.3 gives $\bar{x}$ as a global minimiser of problem $\left(\right.$ LIP $\left._{1}\right)$.

\section{Applications to discrete minimisation problems}

In this section, we will apply the technique, described in Section 3, to a smooth minimisation problem with discrete constraints:

$$
\min _{x \in \mathbb{R}^{n}} f(x) \text { s.t. }\left\{\begin{array}{l}
F_{0}+\sum_{i=1}^{n} x_{i} F_{i} \succeq 0, \\
x \in \prod_{i=1}^{n}\left\{u_{i}, v_{i}\right\}
\end{array}\right.
$$

where $f: \mathbb{R}^{n} \rightarrow \mathbb{R}$ is a twice continuously differentiable function on an open set containing $\Delta:=\prod_{i=1}^{n}\left[u_{i}, v_{i}\right]$ and $F_{i} \in S^{m}, i=0, \ldots, n$. Let $C:=\prod_{i=1}^{n}\left\{u_{i}, v_{i}\right\}$. This model problem covers important optimisation problems with bivalent constraints [10], where $x_{i}=-1$ or $x_{i}=+1$ or binary constraints [2] where $x_{i}=0$ or $x_{i}=1$. Such discrete problems include the quadratic assignment problem [10] and the max-cut problem [2].

In this section, we apply the results of the previous section to derive sufficient global optimality conditions for the discrete model problem $\left(\mathrm{LMIP}_{2}\right)$ by examining a continuous relaxation of the discrete constraints.

THEOREM 4.1. Let $\bar{x} \in \Gamma \cap C$. If there exists $M \succeq 0$ such that $\operatorname{Tr}[M F(\bar{x})]=0$ and

$$
-\frac{1}{2} G(v-u)+\tilde{X}\left(\nabla f(\bar{x})-\hat{F}^{*}(M)\right) \leq 0,
$$

then $\bar{x}$ is a global minimiser of problem $\left(\mathrm{LMIP}_{2}\right)$.

PROOF. Let $g(x):=\frac{1}{2} x^{T} G x+\left(\nabla f(\bar{x})-G \bar{x}-\hat{F}^{*}(M)\right)^{T} x, x \in \Delta:=\prod_{i=1}^{n}\left[u_{i}, v_{i}\right]$. Lemma 3.1 yields that $f(x)-f(\bar{x}) \geq g(x)-g(\bar{x})$ for all $x \in \Gamma \cap C$ as $D \supset \Gamma \cap C$, where $D=\Gamma \cap \Delta$. The conclusion will follow if we show that $\bar{x}$ is a minimiser of $g$ over $C=\prod_{i=1}^{n}\left\{u_{i}, v_{i}\right\}$, which means that for all $x \in \prod_{i=1}^{n}\left\{u_{i}, v_{i}\right\}$,

$$
g(x)-g(\bar{x})=\sum_{i=1}^{n} \frac{\alpha_{i}}{2}\left(x_{i}-\bar{x}_{i}\right)^{2}+\sum_{i=1}^{n}\left(\nabla f(\bar{x})-\hat{F}^{*}(M)\right)_{i}\left(x_{i}-\bar{x}_{i}\right) \geq 0 .
$$

By Proposition 2.2, $\bar{x}$ is a global minimiser of $g$ over $C$ if and only if for each $i=1, \ldots, n, \tilde{x}_{i}\left(d_{i}+\alpha_{i} \bar{x}_{i}\right)-\frac{1}{2} \alpha_{i}\left(v_{i}-u_{i}\right) \leq 0$, where $d_{i}=(\nabla f(\bar{x}))_{i}-\alpha_{i} \bar{x}_{i}-\left(\hat{F}^{*}(M)\right)_{i}$. By hypothesis, (4.1) holds, and so, (4.2) holds. Hence $\bar{x}$ is a global minimiser of $g$ over $C$. 
EXAMPLE 3. Consider the following smooth minimisation problem:

$$
\min _{x \in \mathbb{R}^{2}} f(x)=x_{1}^{3}+x_{2}^{3}-x_{1} x_{2} \quad \text { s.t. } \quad\left\{\begin{array}{l}
F_{0}+\sum_{i=1}^{2} x_{i} F_{i} \geq 0, \\
x \in \prod_{i=1}^{2}\{1,2\},
\end{array}\right.
$$

where

$$
F_{0}=\left(\begin{array}{lll}
3 & 1 & 0 \\
1 & 1 & 0 \\
0 & 0 & 1
\end{array}\right), \quad F_{1}=\left(\begin{array}{lll}
0 & 1 & 0 \\
1 & 0 & 0 \\
0 & 0 & 1
\end{array}\right) \quad \text { and } \quad F_{2}=\left(\begin{array}{lll}
1 & 0 & 0 \\
0 & 0 & 0 \\
0 & 0 & 0
\end{array}\right) \text {. }
$$

It is easy to check that the feasible set is $\{(1,1),(1,2)\}$. Let $\bar{x}=\left(\bar{x}_{1}, \bar{x}_{2}\right)=(1,1)$. Let $z \in \Delta$. Then

$$
\begin{aligned}
F(\bar{x}) & =\left(\begin{array}{ccc}
4 & 2 & 0 \\
2 & 1 & 0 \\
0 & 0 & 1
\end{array}\right), \quad \nabla f(\bar{x})=(2,2)^{T} \\
\nabla^{2} f(z) & =\left(\begin{array}{cc}
6 z_{1} & -1 \\
-1 & 6 z_{2}
\end{array}\right) \quad \text { and } \quad G=\left(\begin{array}{ll}
5 & 0 \\
0 & 5
\end{array}\right) .
\end{aligned}
$$

Taking

$$
M=\left(\begin{array}{ccc}
1 & -2 & 0 \\
-2 & 4 & 0 \\
0 & 0 & 0
\end{array}\right)
$$

we see that $M \in S_{+}^{3}, \operatorname{Tr}[M F(\bar{x})]=0$ and $\hat{F}^{*}(M)=\left(\operatorname{Tr}\left[M F_{1}\right], \operatorname{Tr}\left[M F_{2}\right]\right)^{T}=$ $(-4,1)^{T}$. Now,

$$
\begin{aligned}
-\frac{1}{2} G(v-u)+\tilde{X}\left(\nabla f(\bar{x})-\hat{F}^{*}(M)\right) & =-\frac{1}{2}\left(\begin{array}{ll}
5 & 0 \\
0 & 5
\end{array}\right)\left(\begin{array}{l}
1 \\
1
\end{array}\right)+\left(\begin{array}{cc}
-1 & 0 \\
0 & -1
\end{array}\right)\left(\begin{array}{l}
6 \\
1
\end{array}\right) \\
& =\left(\begin{array}{c}
-17 / 2 \\
-7 / 2
\end{array}\right) .
\end{aligned}
$$

Hence (4.1) holds for (E3) at $\bar{x}=(1,1)$, which is a global minimiser of (E3).

Finally, consider the problem

$$
\min _{x \in \mathbb{R}^{n}} f(x) \text { s.t. }\left\{\begin{array}{l}
b_{0}+B x \geq 0 \\
x \in \prod_{i=1}^{n}\left\{u_{i}, v_{i}\right\}
\end{array}\right.
$$

where $B=\left(b_{i j}\right)_{m \times n}$ is an $m \times n$ matrix and $b_{0}=\left(b_{01}, \ldots, b_{0 m}\right)^{T}$.

COROLlaRY 4.2. Let $\bar{x} \in D$. If there exists $\lambda \in \mathbb{R}^{m}, \lambda \geq 0$ such that $\lambda^{T}\left(b_{0}+B x\right)=$ 0 such that

$$
-\frac{1}{2} G(v-u)+\tilde{X}(\nabla f(\bar{x})-B \lambda) \leq 0,
$$

then $\bar{x}$ is a global minimiser of problem $\left(\mathrm{LIP}_{2}\right)$. 
PROOF. For each $i=0, \ldots, n$, let $F_{i}=\operatorname{diag}\left(b_{i 1}, \ldots, b_{i m}\right)$. Let $M=\operatorname{diag}(\lambda)$. Then $\hat{F}^{*}(M)=B \lambda$. Applying Theorem 4.1 gives $\bar{x}$ as a global minimiser of problem $\left(\mathrm{LIP}_{2}\right)$.

\section{Acknowledgements}

The authors are grateful to Dr Bruce Craven for his valuable suggestions and constructive comments which have contributed to the final preparation of the paper. This work was partially supported by KOSEF International Cooperation Research Program (Grant No. F01-2005-000-10007-0) and by the Australian Research Council Linkage Program. The work of N. Q. Huy was carried out while he was supported by KOSEF Postdoctoral Fellowship Program.

\section{References}

[1] I. G. Akrotirianakis and C. A. Floudas, "Computational experience with a new class of convex underestimators: Box-constrained NLP problems", J. Global Optim. 29 (2004) 249-264.

[2] A. Beck and M. Teboulle, "Global optimality conditions for quadratic optimization problems with binary constraints", SIAM J. Optim. 11 (2000) 179-188.

[3] A. Ben-Tal and A. Nemirovski, Lectures on Modern Convex Optimization: Analysis, Algorithms and Engineering Applications (SIAM-MPS, Philadelphia, 2000).

[4] G. Dahl, "A note on diagonally dominant matrices", Linear Algebra Appl. 317 (2000) 217-224.

[5] C. A. Floudas and V. Visweswaran, "Quadratic optimization", in Handbook of Global Optimization (eds. R. Horst and P. M. Pardalos), (Kluwer Academic Publishers, Dordrecht, 1995) 217-269.

[6] C. Helmberg, "Semidefinite programming", European J. Oper. Res. 137 (2002) 461-482.

[7] J. B. Hiriart-Urruty, "Conditions for global optimality 2", J. Global Optim. 13 (1998) 349-367.

[8] J. B. Hiriart-Urruty, "Global optimality conditions in maximizing a convex quadratic function under convex quadratic constraints", J. Global Optim. 21 (2001) 445-455.

[9] V. Jeyakumar, A. M. Rubinov and Z. Y. Wu, "Sufficient global optimality conditions for nonconvex quadratic minimization problems with box constraints", Applied Mathematics Research Report AMR05/20, University of New South Wales, Australia, to appear in J. Global Optim.

[10] M. C. Pinar, "Sufficient global optimality conditions for bivalent quadratic optimization", J. Optim. Theory Appl. 122 (2004) 433-440.

[11] M. J. Todd, "Semidefinite optimization", Acta Numerica 10 (2001) 515-560.

[12] H. Wolkowicz, R. Saigal and L. Vandenberghe, Handbook of semidefinite programming, International Series in Operations Research and Management Science 27 (Kluwer Academic Publishers, Dordrecht, 2000). 